\title{
THE USE OF AN ORAL SALT LOAD FOLLOWED BY FUROSEMIDE IN THE TREATMENT OF EUVOLEMIC SIADH-INDUCE HYPONATREMIA
}

\author{
A. Ortolá Buigues¹, I. Crespo Hernández¹, T. Ruiz Gracia¹, E. Gómez Hoyos², M. Cuesta Hernández², R. Penso Espinoza ${ }^{1}$ \\ A. Amengual Galbarte ${ }^{1}$, AL. Calle Pascual ${ }^{1}$, P. de Miguel Novoa ${ }^{1}$, I. Runkle de la Vega ${ }^{1}$
}

${ }^{1}$ Hospital Clínico San Carlos, Madrid, Spain.

${ }^{3}$ Beaumont Hospital. Dublin, Ireland.

\section{Introduction}

\section{Material and Methods}

Furosemide can be used in the treatment of SIADH However, to be effective, renal medulla osmolality as reflected in urinary osmolality (UOsm) must be high Sodium is the most important contributer to renal medulla osmolality, which increases following the administration of oral salt. We analyze the use of an oral salt load followed by furosemide for the acute/short-term treatment of euvolemic SIADH hyponatremia.
Retrospective analysis of 9 patients with SIADH-induced hyponatremia and moderate hyponatremic encephalopathy receiving $4-5 \mathrm{~g}$ of oral salt, followed 3 hours later by $20 \mathrm{mg}$ furosemide iv or $40 \mathrm{mg}$ furosemide po (salt-plus-furosemide). Serum sodium levels $(\mathrm{SNa})$, serum potassium (SK), urinary sodium ( $\mathrm{UNa}$ ) were measured at baseline, and 12 to 16 hours following salt administration. All $\mathrm{SNa}$ were corrected for glycemia. Osmolality was measured by In 6/9 patients, a $\mathrm{SNa}$ level was available from 24 hours or less previously. Osmolality was measured by Advanced Instruments Model 3320 osmometer

Variables were compared using Wilcoxon and Mann-Whitney test. Data analysis used SPSS15 software.

\section{Results}

Baseline characteristics:

$4 / 9(44.4 \%)$ were women and 5/9 (65.6\%) were men. Average age was 69.3 (SD 20.7). All patients were clinically euvolemic, with normal ocular pressure and central venous pressure.

Prior to salt-plus-furosemide:

$\mathrm{SNa}$ levels were descending in $5 / 6(83.3 \%)$ patients with a median change of -1.5 [IQR $-2.3-$ $0.25] \mathrm{mmol} / \mathrm{L}$.

Mean 24 hours or less previously SNa was 121 (SD 4.5) $\mathrm{mmol} / \mathrm{L}$

Initial laboratory tests:

Mean SNa was 119 (SD 4.8) mmol/L, mean was SK 4.4 (SD 0.6) mmol/L, median UNa was 54 [IQR:42.5-86] mmol/L, mean POsm was 249 (SD:7.9) mOsm/kg, mean UOsm 449 was (SD 251.2) $\mathrm{mOsm} / \mathrm{kg}$.

Following salt-plus-furosemide:

$\mathrm{SNa}$ rose from 3 to $7 \mathrm{mmol} / \mathrm{L}$, with a median increment of 5 [IQR 4-7] mmol/L, reaching a median $\mathrm{SNa}$ of 124 [IQR:121-127] mmol/L, mean SK 4.1 (SD:0.4) mmol/L, mean UNa 66.4 (SD 24.6) $\mathrm{mmol} / \mathrm{L}$, mean POsm 259 (SD 7.4) mOsm/kg and mean UOsm 370 (SD 151.4) mOsm/kg

The SNa change post salt-plus-furosemide versus the change prior to salt-plus-furosemide was statistically significant $(p=0.027)$. SK descent was also significant $(p=0.017)$.

All blood pressure levels were below $130 / 85 \mathrm{mmHg}$ before and after salt administration
FIGURE 1: Mean SNa evolution following salt-plus-furosemide

125

124

123

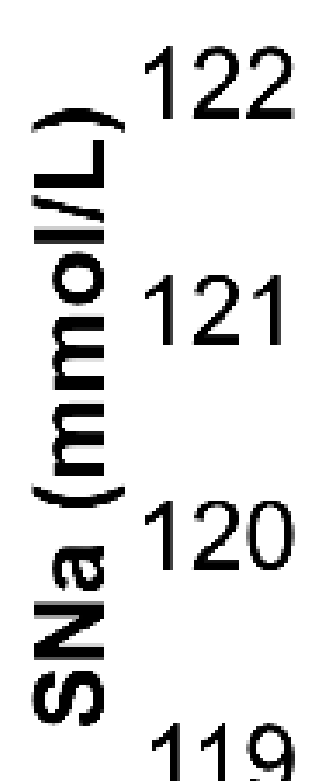

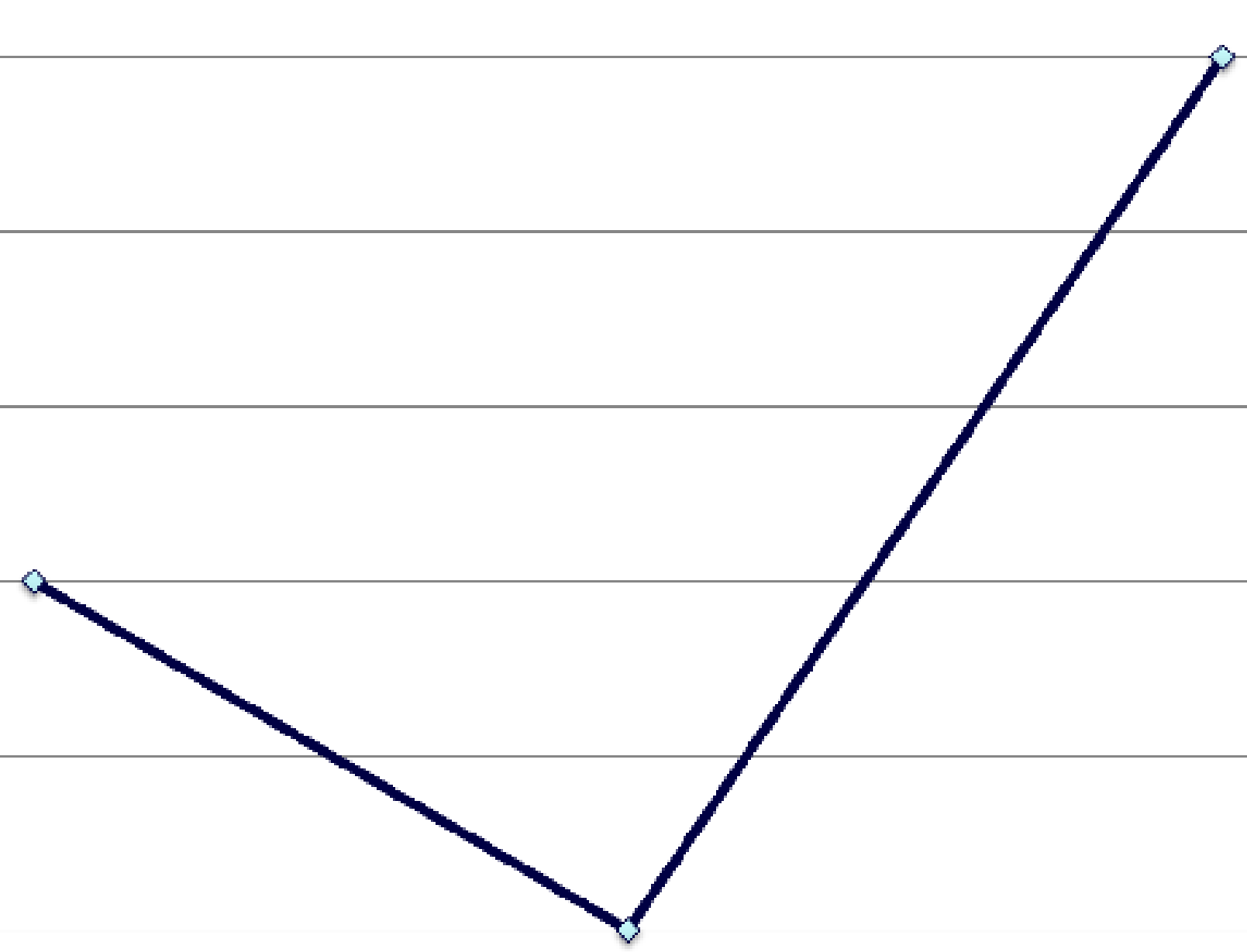

118

117

116 $\begin{array}{ccc}\text { Prior } & \text { Initial } & \text { Following } \\ (12-24 h) & & (12-16 h)\end{array}$
TABLE 1:

Baseline

characteristics

and laboratory

tests evolution of patients

\begin{tabular}{c|c|c|c|} 
& Sex & Age & $\begin{array}{c}\text { Prior } \\
\text { SNa } \\
(\mathrm{mmol} / \mathrm{L})\end{array}$ \\
\cline { 4 - 4 } & & & $\mathbf{1 1 8}$ \\
\hline $1^{* *}$ & Male & 76 & $\mathbf{1 2 5}$ \\
2 & Male & 46 & $\mathbf{1 2 5}$ \\
3 & Male & 27 & $\mathbf{1 2 8}$ \\
4 & Male & 78 & $\mathbf{1 2 0}$ \\
5 & Female & 83 & $\mathbf{1 1 6}$ \\
6 & Female & 67 & $\mathbf{1 2 2}$ \\
7 & Female & 71 & - \\
8 & Female & 82 & - \\
9 & Male & 94 & -
\end{tabular}

** Patient with extremely low prior salt intake. He was diagnosed of SIADH 3 weeks later.
4.2

4.9
245

258

235

245

247
Initial

\begin{tabular}{c|c|c|c|c|}
\hline \multicolumn{5}{|c|}{ Initial } \\
\hline $\begin{array}{c}\mathrm{SNa} \\
\mathrm{mmol} / \mathrm{L})\end{array}$ & $\begin{array}{c}\mathrm{SK} \\
(\mathrm{mmol} / \mathrm{L})\end{array}$ & $\begin{array}{c}\text { POsm } \\
(\mathrm{mOsm} / \mathrm{kg})\end{array}$ & $\begin{array}{c}\text { UNa } \\
(\mathrm{mmol} / \mathrm{L})\end{array}$ & $\begin{array}{c}\text { UOsm } \\
(\mathrm{mmol} / \mathrm{L})\end{array}$ \\
\hline 117 & & 3.5
\end{tabular}

$\begin{array}{lllll}124 & 3.8 & 258 & 53 & 272\end{array}$

$126 \quad 4.1 \quad 254$

$122 \quad 5.2$

$114 \quad 4.2$

$120 \quad 5$

111

116

119
$72 \quad 235$

$54 \quad 643$

$121 \quad 556$

$42 \quad 931$

$69 \quad 593$

$43 \quad 175$

$100 \quad 409$

12-16 hours following salt-plus-furosemide

\begin{tabular}{l|c|c|c|c}
$\mathrm{SNa}$ & $\mathrm{SK}$ & POsm & UNa & UOsm \\
\hline
\end{tabular} $\begin{array}{lllllll}(\mathrm{mmo} / \mathrm{L}) & (\mathrm{mmol} / \mathrm{L}) & (\mathrm{mOsm} / \mathrm{kg}) & (\mathrm{mmol} / \mathrm{L}) & (\mathrm{mmol} / \mathrm{L})\end{array}$ $124 \quad 3.5 \quad 257$

$129 \quad 3.6 \quad 269$

1314263

$127 \quad 4.3 \quad 264$

$121 \quad 4.1 \quad 253$

$127 \quad 4.9 \quad 260$

$114 \quad 4.5 \quad 243$

$123 \quad 4.2 \quad 258$

$124 \quad 4.2 \quad 260$

$82 \quad 441$

$24 \quad 194$

$44 \quad 577$

$60 \quad 330$

$86 \quad 584$

$82 \quad 248$

$87 \quad 233$

233

\section{Conclusions}

The oral administration of 4-5 $\mathrm{g}$ of salt followed by furosemide can be useful in the acute/short-term treatment of euvolemic SIADHhyponatremia. However, this therapy should not be attempted in severe hyponatremia, since a minimum $4 \mathrm{mmol} / \mathrm{L} \mathrm{SNa}$ rise is not assured 